\title{
Reproducibility of crop surface maps extracted from Unmanned Aerial Vehicle (UAV) derived Digital Surface Maps
}

\author{
Stephen D. Parkes*a, Matthew F. McCabe ${ }^{\mathrm{a}}$, Samir K. Al-Mashhawari ${ }^{\mathrm{a}}$, Jorge Rosas ${ }^{\mathrm{a}}$ \\ aHydrology, Agriculture and Land Observation, Water Desalination and Reuse Centre, King Abdullah University of \\ Science and Technology, Kingdom of Saudi Arabia, \\ *stephen.parkes@,kaust.edu.sa, https://hydrology.kaust.edu.sa/Pages/Home.aspx
}

\begin{abstract}
Crop height measured from UAVs fitted with commercially available RGB cameras provide an affordable alternative to retrieve field scale high resolution estimates. The study presents an assessment of between flight reproducibility of Crop Surface Maps (CSM) extracted from Digital Surface Maps (DSM) generated by Structure from Motion (SfM) algorithms. Flights were conducted over a centre pivot irrigation system covered with an alfalfa crop. An important step in calculating the absolute crop height from the UAV derived DSM is determining the height of the underlying terrain. Here we use automatic thresholding techniques applied to RGB vegetation index maps to classify vegetated and soil pixels. From interpolation of classified soil pixels, a terrain map is calculated and subtracted from the DSM. The influence of three different thresholding techniques on CSMs are investigated. Median Alfalfa crop heights determined with the different thresholding methods varied from $18 \mathrm{~cm}$ for $\mathrm{K}$ means thresholding to $13 \mathrm{~cm}$ for Otsu thresholding methods. Otsu thresholding also gave the smallest range of crop heights and $\mathrm{K}$ means thresholding the largest. Reproducibility of median crop heights between flight surveys was $4-6 \mathrm{~cm}$ for all thresholding techniques. For the flight conducted later in the afternoon shadowing caused soil pixels to be classified as vegetation in key locations around the domain, leading to lower crop height estimates. The range of crop heights was similar for both flights using $\mathrm{K}$ means thresholding $(35-36 \mathrm{~cm})$, local minimum thresholding depended on whether raw or normalised RGB intensities were used to calculate vegetation indices $(30-35 \mathrm{~cm})$, while Otsu thresholding had a smaller range of heights and varied most between flights $(26-30 \mathrm{~cm})$. This study showed that crop heights from multiple survey flights are comparable, however, they were dependent on the thresholding method applied to classify soil pixels and the time of day the flight was conducted.
\end{abstract}

Keywords: UAV, Crop Height, Vegetation Index, Alfalfa

\section{INTRODUCTION}

Recent introduction of miniaturised sensors that can be deployed on board novel UAVs have the potential to drive advances in precision agriculture. These developments can provide ultra-high centimetre resolution and have potential to improve temporal resolution compared to satellite based remote sensing. Deployment of a range of sensing systems on board small UAVs have been examined to investigate crop fraction ${ }^{1}$, vegetation properties using hyperspectral ${ }^{2,3}$ and multispectral sensors ${ }^{4}$, weed detection ${ }^{5,6}$, estimating evaporative fluxes using thermal sensors ${ }^{7}$, and crop height/growth monitoring using LIDAR sensors ${ }^{8,9}$. While many of these sensors are relatively expensive, crop height and growth as well as vegetation information have been retrieved using more affordable commercial digital camera' ${ }^{10-13}$. 
Monitoring vegetation height is important for monitoring crop health and growth, yet before introduction of UAV based methodologies, monitoring crop height was time consuming and difficult to provide accurate field scale measurements. While tractor or harvester based deployment of sensors to monitor vegetation height can increase spatial coverage relative to manual techniques, these can be restricted to roadways around a crop or to the end of a cropping cycle. UAV based monitoring of crop growth can therefore improve both spatial and temporal coverage and resolution.

Structure for Motion (SfM) algorithms that use RGB imagery collected onboard UAV's with a high amount of overlap can be used to reconstruct agricultural Digital Surface Maps (DSM). Automated techniques have been developed which identify key points observed in multiple images and can be used for automated aerial triangulation and camera orientation adjustments. Commercially available software has been developed to process imagery collected from UAV's, which when combined with appropriate georeferences can provide accurate DSM's and have been applied to assessing vegetation height and growth ${ }^{11,14}$.

Application of UAV RGB imagery for retrieving crop height has been demonstrated for a range of crops. Zarco-Tejada et $a l^{14}$ in an Olive plantation showed good agreement with manually measured tree heights. Geipel et al ${ }^{11}$ surveyed a maize crop over a growing cycle to determine crop yield. Grenzdörffer ${ }^{15}$ determined that UAV based retrievals underestimated wheat, rapeseed and maize heights compared to conventional techniques. Bendig et al ${ }^{10}$ applied UAV retrievals to rice fields with different $\mathrm{N}$-fertiliser loadings. Bendig et $a l^{3}$ used RGB vegetation indices and SfM crop height estimates to monitor changes in biomass of a barley crop. While these and others have demonstrated some possible applications and in some cases assessed accuracy, reproducibility of crop height retrievals across multiple flights has not been assessed. To improve understanding of uncertainty associated with UAV crop height estimates it is important to determine reproducibility, especially under different meteorological and lighting conditions.

Extracting the absolute height of a crop from a DSM is problematic and can lead to inaccuracies, especially for dense crop covers where identifying the ground surface can be difficult. Different techniques have been applied to extract terrain elevation from DSMs including object-based feature recognition ${ }^{5,11,14}$ and height thresholding ${ }^{15}$ approaches. Height thresholding was shown to underestimate crop height and object-based feature recognition is difficult to apply for crops without clearly defined features. Applying thresholding techniques to RGB vegetation indices to classify soil and vegetation pixels has been successfully applied to images of single plants ${ }^{16}$ or terrestrial images ${ }^{17}$, and could be applied to UAV imagery. This technique can be easily automated for the large datasets typically collected and has potential to be applied to a wide range of cropping systems. However, the reproducibility of using different automated thresholding techniques requires evaluation.

The main aim of this work was to determine the reproducibility of CSMs calculated from 2 flights conducted over an alfalfa covered centre pivot irrigation system in Saudi Arabia ${ }^{18}$. To calculate a CSM from the DSM, a Digital Terrain Map (DTM) is constructed by interpolation between classified soil pixels. Three thresholding methods are applied to the Excess Green vegetation index to automate classification of soil and vegetation pixels. The effect of these thresholding methods on the absolute crop height estimates and their reproducibility across flights is assessed.

\section{METHODS}

\subsection{Site description}

A UAV flight campaign was conducted at the commercial Tawdeehiya Arable farm in the Al-Kharj region of central Saudi Arabia (Figure 1). The site operates 47 centre pivot irrigation systems under a range of different crops (Alfalfa, Carrots, Maize, Potato, Rhodes Grass and Spring Onion). The region is classified as a Hot Desert Climate with summer daytime maximum temperatures averaging $43^{\circ} \mathrm{C}$ and winter $26^{\circ} \mathrm{C}^{19}$. UAV surveys were conducted under clear skies on the 27 th January 2016 over an alfalfa crop 4 days before harvest. 

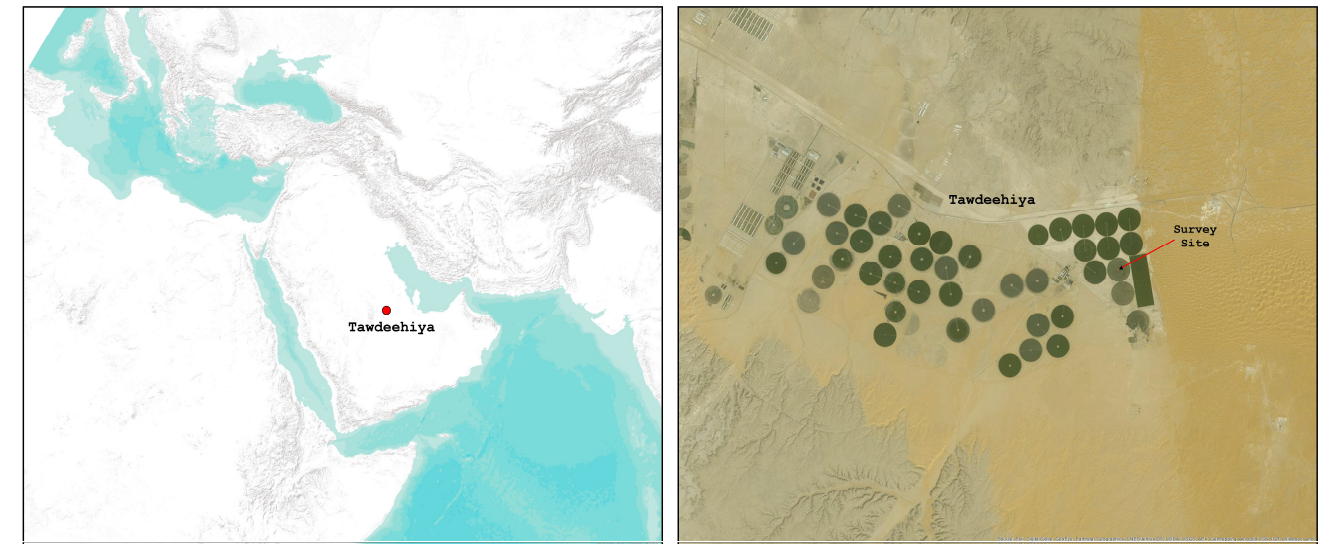

Figure 1: Location of Tawdeehiya Arable Farm in Saudi Arabia where crop height surveys were conducted. The Survey Site is the centre pivot over which flights were performed.

\subsection{UAV flights and camera}

The UAV used in this study was an aileron controlled fixed wing system carrying a 24MP Sony Nex-7 (sensor dimensions were $23.5 \times 15.6 \mathrm{~mm}$ ) with $20 \mathrm{~mm} \mathrm{f} / 2.8$ Alpha E mount lens. To investigate reproducibility of CSMs between surveys, two flights were performed at 14:15 and 16:30 of the 27th January 2016. Flights details and calculated parameters are shown in Table 1, indicating the only major difference between the two flights was the sun angle. Flights were planned with large overlap between images, photos were taken every second for a $84 \%$ frontal overlap and adjacent transects were separated by $60 \mathrm{~m}$, giving 14 transects per flight and $57 \%$ side overlap. Flight routes and locations where photos were collected are shown in Figure $2 \mathrm{a}$ and $\mathrm{b}$.

\subsection{Georeferencing images}

To enable accurate comparison of CSMs determined from separate flights, they must be accurately georeferenced. For this purpose 7 Ground Control Points (GCP) were deployed around a central area of the centre pivot (see Figure 3 for positioning). GCP positions were accurately measured with an RTK-GPS system (Leica Viva GS10 Base and Rover, Leica Geosystems, St. Gallen, Switzerland), which provides horizontal and vertical accuracy of 0.8 and $1.5 \mathrm{~cm}$. Analysis in the present study focuses upon the subset of the survey domain (Figure 3) where GCPs are within a reasonable proximity to all points within the domain.

Table 1: Details for 2 flights conducted.

\begin{tabular}{ccc}
\hline Parameter & Flight 1 & Flight 2 \\
\hline Takeoff Time (LST) & $14: 15$ & $16: 30$ \\
Sun Altitude & $36.6^{\circ}$ & $12.4^{\circ}$ \\
Average Flight Altitude (m) & 121.6 & 121.9 \\
Average Ground Speed (m/s) & 15.3 & 14.9 \\
Footprint (frontal x side $-\mathrm{m})$ & 931. x 140.6 & $93.3 \times 140.6$ \\
Frontal Overlap (\%) & 84 & 84 \\
Side Overlap (\%) & 57 & 57 \\
Number of Images & 943 & 918 \\
\hline
\end{tabular}

"Estimated from planned distance between adjacent flight paths. 


\section{a) Flight 1}

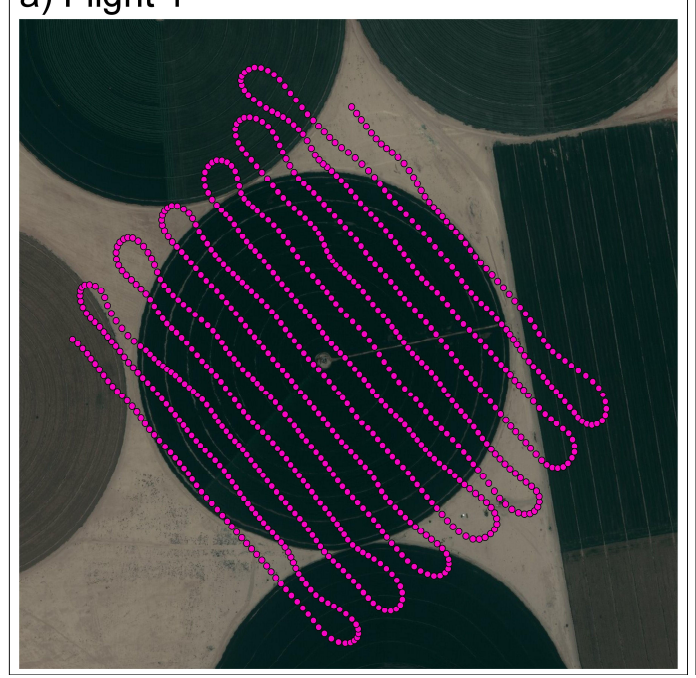

\section{b) Flight 2}

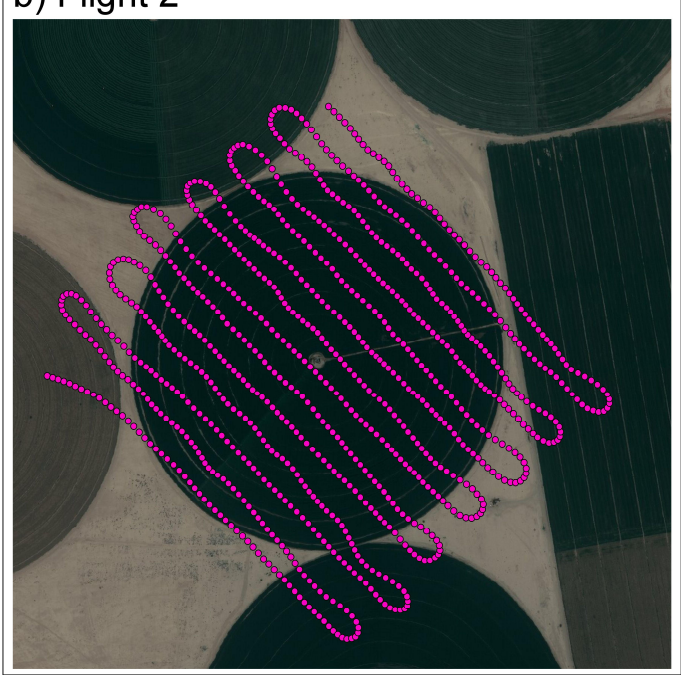

Figure 2: Flight routes and locations where photos were taken (pink circles) for a) flight1 and b) flight 2.

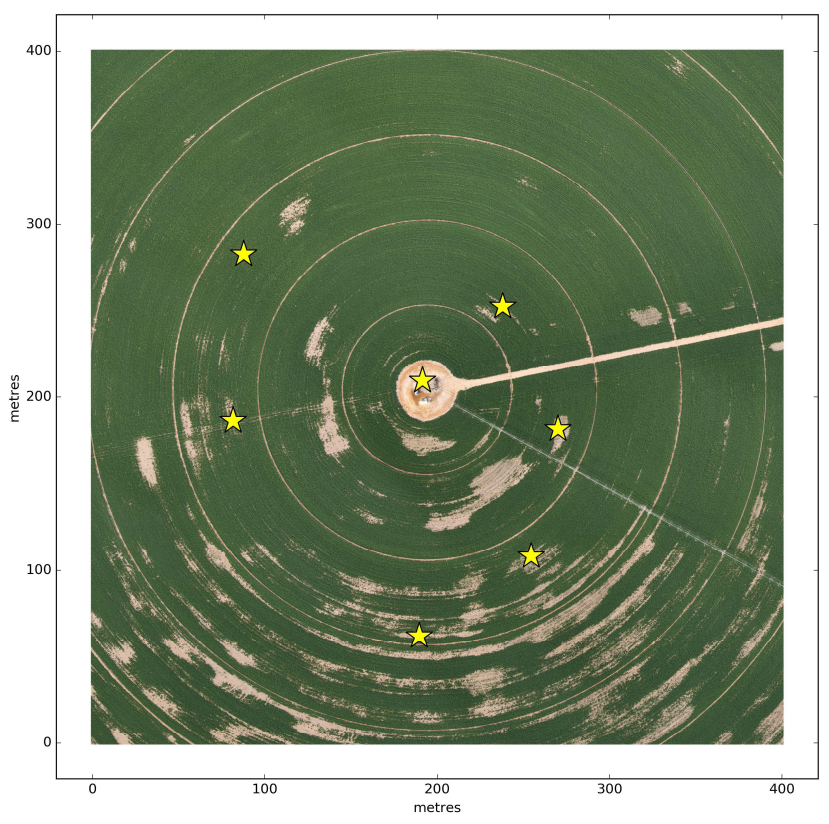

Figure 3: Orthomosaic of the analysis area used in this study are shown in c), along with GCP's (yellow stars). The orthomosaic is the mean from the 2 flights. 


\subsection{Workflow for CSM construction}

Workflow to construct CSM's from UAV imagery started by using the Pix4D mapper software package (Lausanne, Switzerland) to construct orthomosaics, DSM and RGB maps. With the ultimate aim of separating soil from vegetated pixels to calculate a DTM, RGB maps were used to calculate the Excess Green $(2 x G-R-B)$ vegetation index. Excess Green was chosen for the present dataset as it provided the clearest bimodal distribution between soil and vegetated pixels (Figure 4). Excess Green maps were calculated from the raw RGB and normalised intensities. Normalising RGB components accounts for changes in illumination between images. The purpose of investigating raw and normalised RGB intensities is that pixel wise normalisation of each colour channel on large high resolution datasets is time consuming, so eliminating this step would reduce processing times.

To classify soil and vegetated pixels, automatic thresholding methods were applied independently to raw and normalised Excess Green. Otsu thresholding ${ }^{20}, \mathrm{~K}$ means clustering calculated with 2 clusters, and the local minimum between the 2 peaks of the Excess Green histogram (referred to as local minimum threshold) were all applied for pixel classification. Figure 4 shows thresholds for raw and normalised RGB intensities and threshold values are shown in Table 2. While raw and normalised Excess Green histograms are similar significantly different thresholds were determined for Otsu and local minimum techniques. For all techniques flight 2 had a lower number of pixels classified as soil.

Table 2: Excess Green thresholds and number of pixels classified as soil calculated using different methods.

\begin{tabular}{ccccc}
\hline Threshold method & $\begin{array}{c}\text { Flight 1 } \\
\text { Threshold }\end{array}$ & $\begin{array}{c}\text { Flight 1 no. Soil } \\
\text { Pixels (x10 }\end{array}$ & $\begin{array}{c}\text { Flight 2 } \\
\text { Threshold }\end{array}$ & $\begin{array}{c}\text { Flight 2 no. Soil } \\
\text { Pixels (x10 }\end{array}$ \\
\hline Otsu - raw RGB & 37.0 & 11.282 & 29.7 & 10.757 \\
Otsu - normalised RG & 39.7 & 10.923 & 36.1 & 9.512 \\
K means - raw RGB & 11.6 & 6.646 & 9.3 & 5.832 \\
K means - normalised RGB & 9.9 & 7.010 & 8.8 & 5.913 \\
Local min. - raw RGB & 21.4 & 8.182 & 12.9 & 6.490 \\
Local min. - normalised RGB & 29.7 & 9.685 & 27.4 & 8.363 \\
\hline
\end{tabular}

${ }^{*}$ Estimated from planned distance between adjacent flight paths.
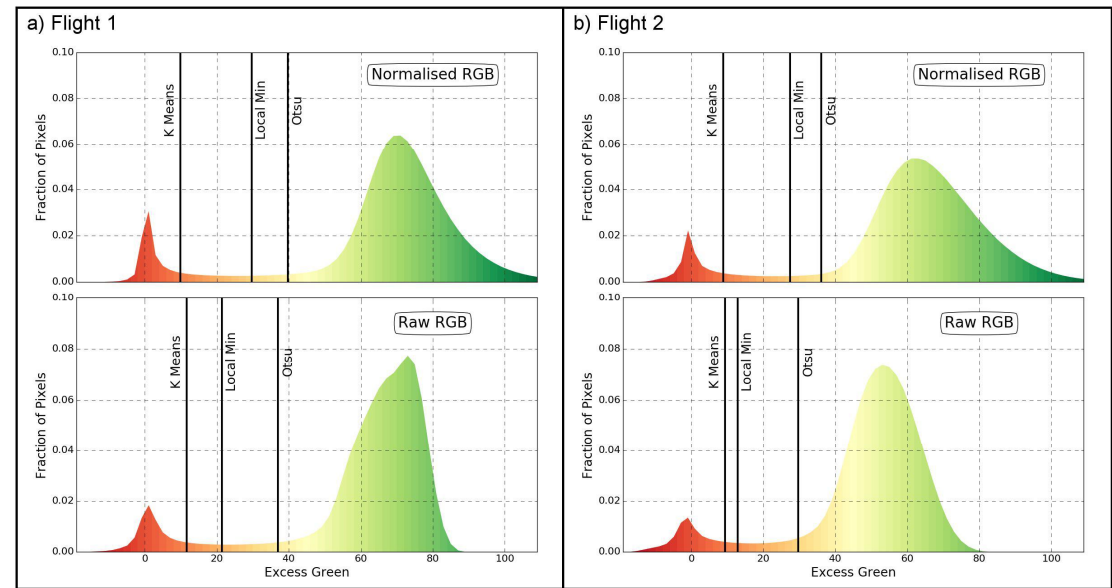

Figure 4: Excess Green histograms for 2 flights showing the different thresholds used to separate soil and vegetated pixels. 
After soil pixels were classified, DTM's were calculated for each flight, RGB processing, and thresholding technique by interpolation using nearest neighbour method. A total of 12 DTM's and therefore CSM's were calculated. Finally, CSM's were calculated for each DTM by subtracting the DTM from the DSM. Each flight was treated individually, so that the difference between CSM's calculated from each flight may be influenced by DSM and DTM differences.

\subsection{Comparison of CSM's}

To determine reproducibility of CSM's between flights, comparisons are made at the pixel scale by calculating the RMSE and bias:

$$
\begin{aligned}
& \text { RMSE }=\sqrt{\sum_{i=1}^{n} \frac{\left(z_{i, 1}-z_{i, 2}\right)^{2}}{n}} \\
& \text { bias }=\sum_{i=1}^{n} \frac{\left(z_{i, 1}-z_{i, 2}\right)}{n}
\end{aligned}
$$

where $n$ is the number of pixels in each dataset, $z_{i, 1}-z_{i, 2}$ is the height difference between flight 1 and 2 for pixel $i$.

\section{RESULTS}

\subsection{Comparison of CSM distributions}

Distribution of crop heights calculated from the 2 flights and different thresholding methods are shown in Figure 5. Crop heights retrieved from UAV based methodologies vary between -9 to $33 \mathrm{~cm}$ ( 5 th to 95 th percentiles). Lowest 5 th percentile heights were observed for Otsu thresholding methods, and highest for K means thresholding. Negative crop heights indicate that DTM's are higher than terrain in some areas of the domain. This could arise from vegetation pixels being classified as soil pixels for the DTM interpolation, consistent with Otsu thresholding having the most negative crop heights and the largest number of pixels classified as soil (Table 2).

Greatest crop heights were observed for K means thresholding (33cm for flight 1 raw RGB intensities) and lowest for Otsu methods ( $18 \mathrm{~cm}$ for flight 2 normalised RGB). The 95th percentile crop heights calculated for different thresholding methods had a weak relationship with the minimum crop height $\left(r^{2}=0.4\right)$, indicating that crop heights were attenuated by a DTM higher than terrain level.

Only small differences were observed between normalised and raw RGB intensity based thresholding. Otsu thresholding shows a small difference between median crop heights when using raw or normalised RGB intensities $(1.5$ and $0.8 \mathrm{~cm}$ for flights 1 and 2), local minimum thresholding had a $3 \mathrm{~cm}$ difference for both flights, and $\mathrm{K}$ means thresholding showed no difference. Thus, at least for K means thresholding, the normalisation of RGB intensities could be by passed.

Larger differences were observed between flights, with flight 1 having higher crop heights regardless of thresholding used. Median crop heights from flight 1 were from $4.7 \mathrm{~cm}$ (K means and local minimum thresholding) to $6.3 \mathrm{~cm}(\mathrm{Otsu}$ thresholding with raw RGB) greater than flight 2. Similar differences were observed for 95th percentile crop heights (4$6.3 \mathrm{~cm})$, while differences between 5 th percentile crop heights were smaller $(2.3-4 \mathrm{~cm})$, which indicates different reasons behind the differences for low and high crop heights. Differences between 95th and 5th percentile heights for K means thresholding was between 35.2 and $36.7 \mathrm{~cm}$ with no clear difference between flights or RGB processing. The local minimum technique showed ranges from 30.5 to $35.2 \mathrm{~cm}$, giving the largest range for raw processing. Otsu thresholding was from 26.6 to $31.3 \mathrm{~cm}$ and gave smaller ranges for flight 2 . 


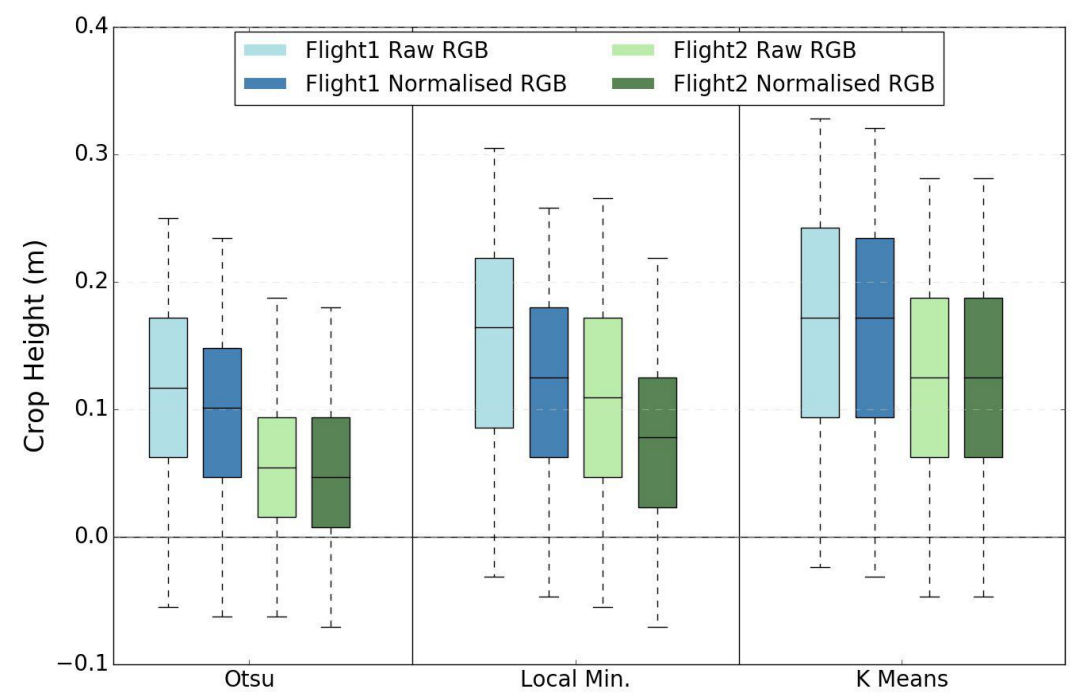

Figure 5: Box plots comparing the crop height from the 2 flights retrieved using different thresholding methods. Whiskers are 5th and 95th percentiles, boxes upper and lower quartiles and medians are the lines in middle of boxes.

\subsection{Between flight reproducibility}

Figure 6 shows the pixel-wise correlations between crop heights retrieved from different flights. Correlations were separated into thresholding and RGB processing methods. For all cases, the data is distributed near the 1:1 line indicating a reasonable agreement between flights, although correlation coefficients are low $\left(\mathrm{r}^{2}\right.$ between 0.37 and 0.47$)$. Percentiles agree, albeit biased towards higher crop heights for flight 1 as already noted. Lower percentiles fall closer to the 1:1 line indicating biases between flights were larger nearer median heights and above. Depending on RGB processing and threshold method, RMSE varied between 8 and $10 \mathrm{~cm}$, and the bias between 3.8 and $5.6 \mathrm{~cm}$. 

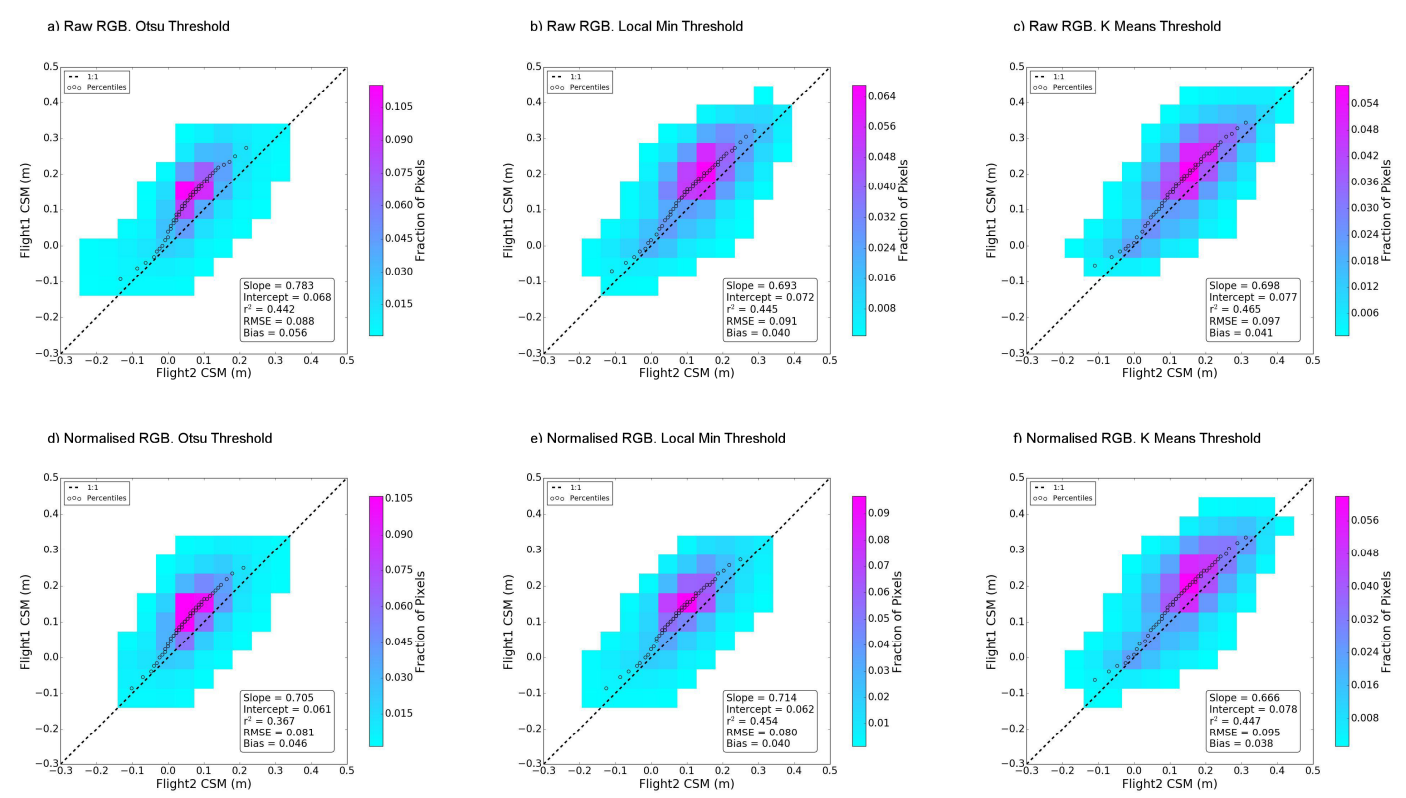

Figure 6: Correlations between CSM's from the 2 flights calculated with different thresholding methods. The colour map shows the concentration of pixels for $5 \mathrm{~cm}$ bins. The dashed curve is the $1: 1$ line, and open circles are the 2 nd to 98th percentiles ( 2 percentile increments). Statistics comparing the 2 flights are shown for each thresholding type.

\section{DISCUSSION}

\subsection{CSM accuracy}

Negative crop height retrievals showed the UAV approach underestimates crop height, with the magnitude depending on thresholding method used to classify soil pixels. In the present study, elevated DTMs are the likely source of underestimated crop heights and observed negative heights. Negative crop heights were probably observed because vegetated pixels were classified as soil causing interpolation between some points with higher elevations than the terrain. The three thresholding methods classified a very different number of pixels as soil. Otsu thresholding classified the largest number of pixels as soil, so probably misclassified the largest number of vegetated pixels and therefore had the lowest CSM. Conversely, K means thresholding had the lowest number of soil pixels classified and highest CSM. In addition to misclassified soil pixels, terrain features under the vegetation where no nearby soil pixels were identified, especially depressions, crop height would be underestimated. For the centre pivot system surveyed here, irrigation boom wheel tracks, access road and central pumping area were raised above the crop bed, if no pixels were defined at crop terrain level adjacent these features, DTM's would be elevated above terrain level. While thresholds could be tuned to avoid misclassification, missed terrain features are probably unavoidable and in more complex terrain may be prohibitive. However, in relatively flat terrain as studied here, reasonable estimates of crop height can still be obtained.

\subsection{CSM reproducibility across flights}

Between flight reproducibility was a major contributor to CSM uncertainty, with similar differences between flights for all thresholding methods or RGB processing. Here we discuss between flight reproducibility focusing on the $\mathrm{K}$ mean thresholding technique as it had the least negative crop heights and therefore is presumed the most accurate estimate. We further focus on CSM's calculated with normalised RGB intensities, as RGB processing had a negligible influence on K means thresholding. 
Contributions to between flight reproducibility arise from the DSM and DTM. Figure 7 shows maps of differences between the CSM, DSM and DTMs determined from the 2 flights. Georeferenced DSMs are in good agreement, with median crop heights for flight $15 \mathrm{~cm}$ lower, while 5 th and 95th percentiles deviated by -16 and $4 \mathrm{~cm}$, respectively. The largest differences occur away from GCPs (see Figure 3), indicating the importance of including an even distribution of GCPs across a domain. Near the GCPs, flight 2 had slightly higher elevations, but only by $5-10 \mathrm{~cm}$.

DSM reproducibility was of similar magnitude to the difference between median crop heights (Figure 5). However, there is no relationship between the difference of CSMs and DSMs (see Figure 7a and b). CSM reproducibility had a similar pattern to DTM reproducibility (Figure 7c) and shows a negative correlation (Figure 8). Comparison of the CSM reproducibility does not reflect spatial variability in crop height (Figure 7d). This all indicates that using the method outlined here, the DTM is the major factor influencing CSM reproducibility between flights.
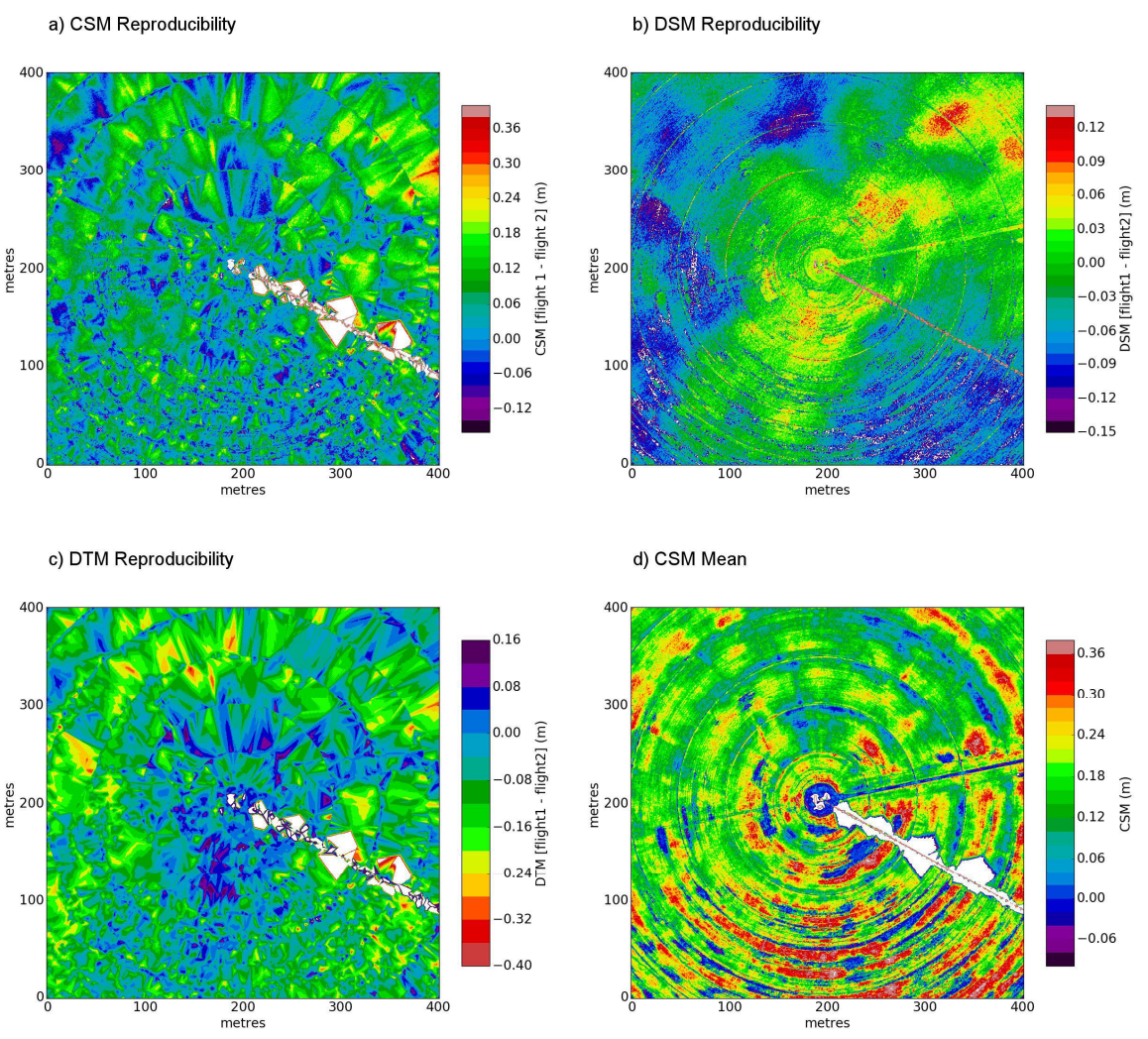

Figure 7: Maps of between flight reproducibility for a) CSM's, b) DSM and c) DTM's. For CSM and DTM's reproducibility was calculated from $\mathrm{K}$ means thresholding on raw RGB intensities. D) shows the mean CSM calculated for the 2 flights with $\mathrm{K}$ means thresholding on raw RGB intensities. 


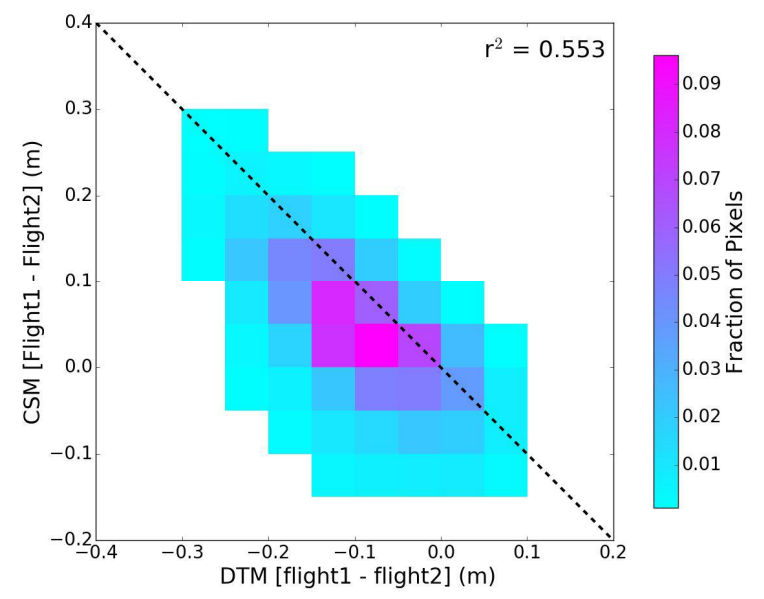

Figure 8: Relationship between CSM and DTM reproducibility.

Lower crop height estimates were determined for flight 2, although less soil pixels were classified, which contrasts to comparisons of crop height a calculated with different thresholding methods. In that case lower numbers of classified soil pixels were related to higher crop heights. This indicates an additional source of error to misclassification of soil pixels contributed to lower crop height estimates for the second flight. Flight 2 was conducted late in the afternoon with lower sun angles casting shadows. Gitelson ${ }^{17}$ observed that dark shadows can lead to misclassification of soil pixels as vegetation. Figure 9 shows the mismatch of soil pixels classified for the 2 flights occurred near features mentioned (see Figure $2 \mathrm{c}$ for reference to features where mismatched classification occurs). Around the whole domain, thin lines of pixels where flight 1 was defined as soil and flight 2 vegetation occur along the edge of the wheel tracks. In general these wheel tracks sloped down to the crop bed, thus leading to a higher DTM for flight 2. By segmenting the orthomosaic to recalculate thresholds, problems with shadowed regions may be overcome. However, lighting conditions can influence crop height biases and flights should be restricted to high sun angles away from sunrise and sunset periods.
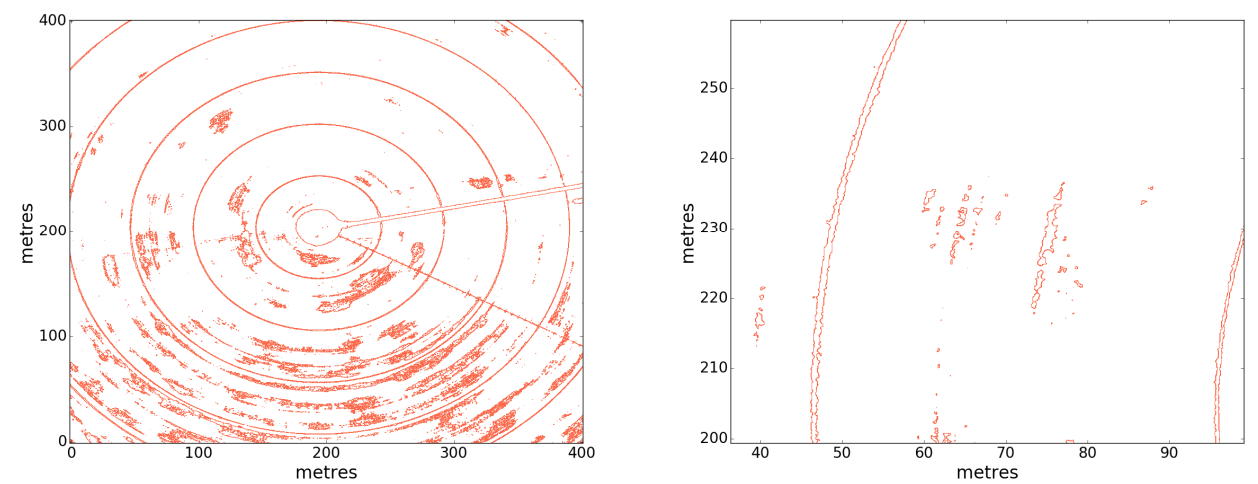

Figure 9: Pixels where flight 1 were defined as soil and flight 2 as vegetation. Right plot shows small inset of the analysed domain and clearly shows the line of pixels on either side of wheel track defined as soil for flight 1 and vegetation for flight 2 . 


\section{CONCLUSIONS}

RGB images collected onboard a UAV were used to construct Digital Surface Maps (DSM) and Crop Surface Maps (CSM) using vegetation indices. The major aim of the study was to investigate between flight reproducibility of CSMs and study the impact of applying different automated thresholding techniques to classify soil pixels for extraction of the elevation of terrain underlying the vegetation. Three different thresholding methods ( $\mathrm{K}$ means, Otsu and histogram local minimum) were used to extract soil pixels from orthomosaics and calculate terrain maps for determining absolute crop heights. The Excess Green vegetation index was used to classify soil and vegetation. The comparison showed that CSMs were sensitive to thresholding method with $\mathrm{K}$ means thresholding providing highest crop heights, and Otsu thresholding the lowest. Comparison of the 2 flights showed similar differences were observed for all thresholding methods and RGB processing, with RMSE of 8-9 $\mathrm{cm}$ and biases of $4-6 \mathrm{~cm}$. Shadows led to lower crop heights determined for the second flight conducted later in the day. This study has shown that determining absolute crop heights from UAV imagery and using vegetation indexes to extract the elevation of underlying terrain shows reasonable reproducibility that is conducive with detecting whole field and within field variability.

\section{REFERENCES}

[1] Torres-Sánchez, J., Peña, J. M., de Castro, A. I.., López-Granados, F., "Multi-temporal mapping of the vegetation fraction in early-season wheat fields using images from UAV," Comput. Electron. Agric. 103, 104113 (2014).

[2] Zarco-Tejada, P. J., González-Dugo, V.., Berni, J. A. J., "Fluorescence, temperature and narrow-band indices acquired from a UAV platform for water stress detection using a micro-hyperspectral imager and a thermal camera," Remote Sens. Environ. 117, 322-337 (2012).

[3] Bendig, J., Yu, K., Aasen, H., Bolten, A., Bennertz, S., Broscheit, J., Gnyp, M. L.., Bareth, G., “Combining UAV-based plant height from crop surface models, visible, and near infrared vegetation indices for biomass monitoring in barley,” Int. J. Appl. Earth Obs. Geoinf. 39, 79-87 (2015).

[4] Berni, J. A. J., Zarco-Tejada, P. J., Suarez, L.., Fereres, E., “Thermal and Narrowband Multispectral Remote Sensing for Vegetation Monitoring From an Unmanned Aerial Vehicle," IEEE Trans. Geosci. Remote Sens. 47(3), 722-738 (2009).

[5] Peña, J. M., Torres-Sánchez, J., de Castro, A. I., Kelly, M.., López-Granados, F., “Weed Mapping in EarlySeason Maize Fields Using Object-Based Analysis of Unmanned Aerial Vehicle (UAV) Images," PLoS One 8(10), e77151, Public Library of Science (2013).

[6] Torres-Sánchez, J., López-Granados, F., De Castro, A. I.., Peña-Barragán, J. M., "Configuration and Specifications of an Unmanned Aerial Vehicle (UAV) for Early Site Specific Weed Management," PLoS One 8(3), e58210, Public Library of Science (2013).

[7] Hoffmann, H., Nieto, H., Jensen, R., Guzinski, R., Zarco-Tejada, P.., Friborg, T., "Estimating evaporation with thermal UAV data and two-source energy balance models," Hydrol. Earth Syst. Sci. 20(2), 697-713, Copernicus Publications (2016)

[8] Wallace, L., Musk, R.., Lucieer, A., “An Assessment of the Repeatability of Automatic Forest Inventory Metrics Derived From UAV-Borne Laser Scanning Data,” IEEE Trans. Geosci. Remote Sens. 52(11), 7160-7169 (2014). 
[9] Wallace, L., Lucieer, A., Watson, C.., Turner, D., "Development of a UAV-LiDAR System with Application to Forest Inventory,” Remote Sens. 4(12), 1519-1543, Molecular Diversity Preservation International (2012).

[10] Bendig, J., Willkomm, M., Tilly, N., Gnyp, M. L., Bennertz, S., Qiang, C., Miao, Y., Lenz-Wiedemann, V. I. S.., Bareth, G., "Very high resolution crop surface models (CSMs) from UAV-based stereo images for rice growth monitoring In Northeast China," ISPRS - Int. Arch. Photogramm. Remote Sens. Spat. Inf. Sci. XL1/W2(September), 45-50 (2013).

[11] Geipel, J., Link, J.., Claupein, W., "Combined Spectral and Spatial Modeling of Corn Yield Based on Aerial Images and Crop Surface Models Acquired with an Unmanned Aircraft System," Remote Sens. 6(11), 1033510355, Multidisciplinary Digital Publishing Institute (2014).

[12] Turner, D., Lucieer, A.., Watson, C., “An Automated Technique for Generating Georectified Mosaics from Ultra-High Resolution Unmanned Aerial Vehicle (UAV) Imagery, Based on Structure from Motion (SfM) Point Clouds," Remote Sens. 4(12), 1392-1410, Molecular Diversity Preservation International (2012).

[13] Wallace, L., Lucieer, A., Malenovský, Z., Turner, D.., Vopěnka, P., “Assessment of Forest Structure Using Two UAV Techniques: A Comparison of Airborne Laser Scanning and Structure from Motion (SfM) Point Clouds," Forests 7(3), 62, Multidisciplinary Digital Publishing Institute (2016).

[14] Zarco-Tejada, P. J., Diaz-Varela, R., Angileri, V.., Loudjani, P., “Tree height quantification using very high resolution imagery acquired from an unmanned aerial vehicle (UAV) and automatic 3D photo-reconstruction methods," Eur. J. Agron. 55, 89-99 (2014).

[15] Grenzdörffer, G. J., "Crop height determination with UAS point clouds,” ISPRS - Int. Arch. Photogramm. Remote Sens. Spat. Inf. Sci. XL-1, 135-140, Copernicus Publications (2014).

[16] Meyer, G. E.., Neto, J. C., "Verification of color vegetation indices for automated crop imaging applications," Comput. Electron. Agric. 63(2), 282-293 (2008).

[17] Gitelson, A. A., Kaufman, Y. J., Stark, R.., Rundquist, D., "Novel algorithms for remote estimation of vegetation fraction," Remote Sens. Environ. 80(1), 76-87 (2002).

[18] Houborg, R.., McCabe, M. F., “Adapting a regularized canopy reflectance model (REGFLEC) for the retrieval challenges of dryland agricultural systems," Remote Sens. Environ. 186, 105-120 (2016).

[19] El Kenawy, A. M., McCabe, M. F., Vicente-Serrano, S. M.., Robaa, S. M., "Recent changes in continentality and aridity conditions over the Middle East and North Africa region, and their association with circulation patterns," Clim. Res. 69(1), 25-43 (2016).

[20] Otsu, N., "Threshold selection method from grey-level histograms," IEEE Trans Syst Man Cybern SMC-9(1), 62-66 (1979). 